\title{
Efficacy and Safety of Topical Dexpanthenol-Containing Spray and Cream in the Recovery of the Skin Integrity Compared with Petroleum Jelly after Dermatologic Aesthetic Procedures
}

\author{
Flávia Alvim Sant'Anna Addor ${ }^{1}$, Maurício Cândido de Souza ${ }^{2}$, Sonja Trapp ${ }^{3}$, Erwan Peltier ${ }^{3}$ (i) \\ and Juliana Machado Canosa $2, *$ (D) \\ Medcin Dermatologia, Osasco 06023-000, Brazil; flavia.addor@medcin.com.br \\ Bayer Brazil, São Paulo 04779-900, Brazil; mauricio.souza1@bayer.com \\ Bayer Switzerland, CH-4052 Basel, Switzerland; sonja.trapp@bayer.com (S.T.); erwan.peltier@bayer.com (E.P.) \\ Correspondence: jmdermato@gmail.com
}

check for

updates

Citation: Addor, F.A.S.; de Souza, M.C.; Trapp, S.; Peltier, E.; Canosa, J.M. Efficacy and Safety of Topical Dexpanthenol-Containing Spray and Cream in the Recovery of the Skin Integrity Compared with Petroleum Jelly after Dermatologic Aesthetic Procedures. Cosmetics 2021, 8, 87 . https://doi.org/10.3390/

cosmetics 8030087

Academic Editor: Nobutomo Ikarash

Received: 2 July 2021

Accepted: 27 August 2021

Published: 10 September 2021

Publisher's Note: MDPI stays neutral with regard to jurisdictional claims in published maps and institutional affiliations.

Copyright: (c) 2021 by the authors. Licensee MDPI, Basel, Switzerland. This article is an open access article distributed under the terms and conditions of the Creative Commons Attribution (CC BY) license (https:// creativecommons.org/licenses/by/ $4.0 /)$.

\begin{abstract}
Moisturizers are commonly prescribed after laser and chemical peel aesthetic procedures, but the evidence regarding their efficacy and safety of such use is scarce. We conducted four singleblind, three-week, controlled studies to evaluate the efficacy and safety of topical Dexpanthenolcontaining products (Bepantol ${ }^{\circledR}$ spray and Bepantol ${ }^{\circledR}$ cream) using petroleum jelly as a positive control. Skin recovery was assessed after four aesthetic procedures: (1) non-ablative facial laser resurfacing, (2) laser depilation on the external genital and inguinal regions, (3) chemical peel on the external genital and inguinal regions, and (4) ablative facial laser resurfacing. Efficacy was assessed through transepidermal water loss (TEWL) combined with clinical assessment of the skin by the investigators and the participants. In studies (1) and (4), the erythema intensity was evaluated by measuring dermal temperature with a thermal imaging camera. Safety was assessed through adverse event reporting and acceptability through a questionnaire. Dexpanthenolcontaining products significantly decreased TEWL and dermal temperature, therefore maintaining skin integrity, promoting its recovery, and reducing erythema. No statistical differences with the positive control were observed. In addition, Dexpanthenol-containing products were well appreciated by the participants from a sensory perspective. These findings suggest that these Dexpanthenolcontaining products are adequate for post-procedural care in aesthetic dermatology.
\end{abstract}

Keywords: dexpanthenol; laser resurfacing; emulsions; skin barrier; skin physiology; wound healing

\section{Introduction}

The use of fractional photothermolysis (FP) laser is increasing in aesthetic clinics for several purposes: rejuvenation, improving the texture of the skin, reduction of small vascular injuries, reduction of wrinkles around the eyes, treatment of melasmas and acne scars, and depilation [1-3]. FP laser creates microscopic columns of thermal damage, interspersing damaged skin tissues (microthermal zones), in areas of perfectly intact skin [2,4]. An FP laser can be ablative or non-ablative, depending on the impact on stratum corneum [2]. Ablative FP has longer wavelengths, in the range of 2940-10,600 nm, destroying the full-thickness of skin. In contrast, non-ablative FP has wavelengths ranging from 1320 to $1927 \mathrm{~nm}$, leaving a functionally and histologically intact stratum corneum [2]. The ablative lasers vaporize the superficial layers of the skin by heating the dermis, usually using pulsed carbon dioxide $\left(\mathrm{CO}_{2}\right)$ laser. However, non-ablative lasers stimulate collagen growth only by creating focal thermal injury within the dermis [5], probably through a mechanism similar to ablative procedures but without the epidermal injury [3]. The healing process and success of laser treatments are enhanced by appropriate postoperative care of the laser-treated skin [5]. 
Other widespread aesthetic procedures that superficially damage the skin barrier are the use of chemical peels (also known as chemexfoliation or chemical exfoliation). Chemical exfoliation is the application of chemical agents, of variable strength, on the skin resulting in a controlled destruction of both epidermis and dermis, to treat hyperpigmentation and acne scars [6-8]. Mandelic acid, a large molecule of an alpha-hydroxy acid, is a commonly used peeling agent [8-11]. After such procedures, adequate care is needed to facilitate the process of natural skin restoration [12].

Currently, there are no guidelines for pre- and post-laser resurfacing treatment or chemical peel. Therefore, the post-procedure regimen is usually based on personal experience of the physician. Maintaining a moist environment is important in the post-procedure period, so the use of "moisturizers" is commonly recommended to maintain the skin surface clean and lubricated. However there is no specific recommendation regarding the physical or chemical attributes of the moisturizer [13].

Dexpanthenol or Pro-vitamin B5 is a well-known ingredient in the skincare industry. Because of its hygroscopic properties, it promotes the retention of moisture, thereby improves skin hydration. Moreover, Dexpanthenol is readily absorbed and rapidly converted enzymatically to pantothenic acid, a constituent of coenzyme A, which catalyzes the synthesis of fatty acids and sphingolipids, important for the structure of lipid layers in stratum corneum. Finally, even though the mechanisms by which Dexpanthenol restores and protects skin barrier function have not been fully elucidated, it has been suggested that Dexpanthenol promotes epidermal regeneration by enhancing epidermal differentiation and lipid synthesis [14-16]. This molecule is a moisturizer with barrier-improving properties and a wound-healing agent. Such properties are beneficial for subjects in need of skin care and/or wound healing.

Therefore, Dexpanthenol-containing preparations stand as an alternative to petroleum jelly [17], which forms a thick occlusive protective layer with the objective of water loss prevention [18]. Several publications, as well as the manufacturers of some $\mathrm{CO}_{2}$ laser devices, recommend the use of petroleum jelly to protect skin tissue from exposure to air until it is completely healed $[13,17,19,20]$.

Although scientific literature on using petroleum jelly as a moisturizer is scarce, it is widely used for wound healing, especially after minor surgical procedures. Its main properties are occlusion, thereby blocking transepidermal water loss and keeping the skin moisturized $[18,21]$.

Beyond product efficacy and safety, features of post-procedural care, such as acceptability, perceived efficacy, pleasantness, and calming effect, could help increase patient compliance with product usage.

To examine the benefits of using Dexpanthenol-containing products as skincare following aesthetic interventions, we have compared the efficacy and the safety of Dexpanthenolcontaining preparations (spray and cream) versus petroleum jelly in the recovery of the skin after four types of dermatological aesthetic procedures: non-ablative and ablative facial laser resurfacing, laser depilation, and chemical peel with mandelic acid.

\section{Methods}

\subsection{Study Design and Participants}

We conducted four single-blind, within-subject comparison, controlled studies to evaluate the efficacy and safety of topical Dexpanthenol-containing products (Bepantol ${ }^{\circledR}$ spray and Bepantol ${ }^{\circledR}$ cream) in healthy adult females with Fitzpatrick phototypes I or II. The status of their skin was assessed after one of the following aesthetic procedures: (1) non-ablative facial laser resurfacing, (2) laser depilation on the external genital and inguinal region, (3) chemical peel with mandelic acid 20\% (in non-ionic gel) on the inguinal/external genital region, and (4) ablative facial laser resurfacing. The compositions of Dexpanthenol containing spray and cream are described at the end of the article.

The studies on laser resurfacing included women aged 30-60 years old and the studies on depilation and chemical peel included women from 18 to 60 years old. For the non- 
ablative laser, we used Genesis ${ }^{\circledR}$ (ND; YAG laser, wavelenght $1064 \mathrm{~nm}$, fluence $14 \mathrm{~J} / \mathrm{cm}^{2}$, exposure time 0.3 microseconds, power $9 \mathrm{~Hz}$ ), and the ablative laser was performed with Erbium YAG Laser (wavelenght $2940 \mathrm{~nm}$, short pulse, laser output $2.5 \mathrm{~J} / \mathrm{cm}^{2}$, and pulse duration 250 microseconds).

All the procedures were bilateral, with each side randomized to receive either Dexpanthenol-containing product or control (petroleum jelly). Petroleum jelly was chosen as the positive control product because of its inert nature as a physical barrier $[17,18]$. Dexpanthenol-containing spray, a water-based product, was used after non-ablative laser resurfacing, laser depilation, and chemical peel, and Dexpanthenol-containing cream was used after ablative facial laser.

The aesthetic procedures were performed on Day 1, followed by the initial application of the test products. The first application was given in the clinic while instructing the patients for the subsequent applications to be performed at home twice daily, preferably after a bath or local cleansing, until day 21. Table 1 summarizes the design of each study.

Table 1. Study designs.

\begin{tabular}{|c|c|c|c|c|c|c|}
\hline Study & Procedure & Body Region & Test Products & Primary Outcome & Secondary Outcomes & $\begin{array}{c}\text { Time of } \\
\text { Assessment }\end{array}$ \\
\hline 1 & $\begin{array}{l}\text { Non-ablative laser } \\
\text { resurfacing }\end{array}$ & Face & $\begin{array}{c}\text { Dexpanthenol- } \\
\text { containing spray } \\
\text { vs. Petroleum jelly }\end{array}$ & $\begin{array}{c}\text { TEWL; } \\
\text { Reduction of } \\
\text { erythema * }\end{array}$ & $\begin{array}{l}\text { Clinical efficacy assessed } \\
\text { by dermatologists, } \\
\text { Efficacy perceived by subjects, } \\
\text { Product safety, tolerability, } \\
\text { and acceptability }\end{array}$ & $\begin{array}{c}\text { T0, } \\
\text { T1, T48, D7, D14, } \\
\text { and D21 }\end{array}$ \\
\hline 2 & Laser depilation & $\begin{array}{l}\text { External genital and } \\
\text { inguinal areas }\end{array}$ & $\begin{array}{c}\text { Dexpanthenol- } \\
\text { containing spray } \\
\text { vs. Petroleum jelly }\end{array}$ & TEWL & $\begin{array}{l}\text { Clinical efficacy assessed } \\
\text { by dermatologists, } \\
\text { Efficacy perceived by subjects, } \\
\text { Product safety, tolerability, } \\
\text { and acceptability }\end{array}$ & $\begin{array}{c}\mathrm{T} 0 \\
\mathrm{~T} 1, \mathrm{~T} 48, \mathrm{D} 7, \text { and } \\
\mathrm{D} 21\end{array}$ \\
\hline 3 & $\begin{array}{l}\text { Chemical peel with } \\
\text { mandelic acid } 20 \%\end{array}$ & $\begin{array}{l}\text { External genital and } \\
\text { inguinal areas }\end{array}$ & $\begin{array}{c}\text { Dexpanthenol- } \\
\text { containing spray } \\
\text { vs. Petroleum jelly }\end{array}$ & TEWL & $\begin{array}{l}\text { Clinical efficacy assessed } \\
\text { by dermatologists } \\
\text { Efficacy perceived by subjects } \\
\text { Product safety, tolerability, } \\
\text { and acceptability }\end{array}$ & $\begin{array}{l}\text { T0, T1, T48, D7, D14, } \\
\text { and D21 }\end{array}$ \\
\hline 4 & $\begin{array}{l}\text { Ablative laser } \\
\text { resurfacing }\end{array}$ & Face & $\begin{array}{l}\text { Dexpanthenol- } \\
\text { containing } \\
\text { emollient cream } \\
\text { vs. Petroleum jelly }\end{array}$ & $\begin{array}{c}\text { TEWL; } \\
\text { Reduction of } \\
\text { erythema * }\end{array}$ & $\begin{array}{l}\text { Clinical efficacy assessed } \\
\text { by dermatologists } \\
\text { Efficacy perceived by subjects } \\
\text { Product safety, tolerability, } \\
\text { and acceptability }\end{array}$ & $\begin{array}{l}\mathrm{T} 0, \mathrm{~T} 1, \mathrm{~T} 48, \mathrm{D} 7, \mathrm{D} 14, \\
\text { and D21 }\end{array}$ \\
\hline
\end{tabular}

T0: initial assessment; T1: immediately after the procedure and before the application of the products (Studies 2 and 3 ) and $1 \mathrm{~h}$ after the procedure and before the application of the products (Studies 1 and 4). T48: after 48 h. D7, D14 and D21: Day 7, Day 14 and D21. TEWL: transepidermal water loss; ${ }^{*}$ measures of dermal temperature by the thermographic camera (FLIR T530).

\subsection{Primary Outcomes}

The primary outcome was the efficacy of Dexpanthenol-containing products in helping the recovery of the skin barrier integrity, assessed after each procedure through the TEWL (transepidermal water loss) measured with a Tewameter ${ }^{\circledR}$ MT 300 (Courage + Khazaka).

TEWL is a validated measure of skin barrier function [22] which was assessed at as baseline (T1) prior to application of test products (Figure 1). T1 was defined as either immediately (in studies (2) and (3)) or one hour after the procedure (in studies (1) and (4), facial laser resurfacing). Thereafter TEWL was assessed after $48 \mathrm{~h}$ (T48), and on the 7th, 14th and 21st days (D7, D14 and D21), except for study (2) (laser depilation), which did not include the assessment on D14.

Reduction of erythema was a primary outcome only for laser resurfacing (studies (1) and (4)), assessed with measures of dermal temperature by a thermal imaging camera (FLIR T530).

Before any assessment, the participants were asked to stay in a room with controlled temperature and humidity $\left(\mathrm{T}=20 \pm 2{ }^{\circ} \mathrm{C}\right.$ and $\mathrm{H}=50 \pm 5 \%$ ) for $20 \mathrm{~min}$. 

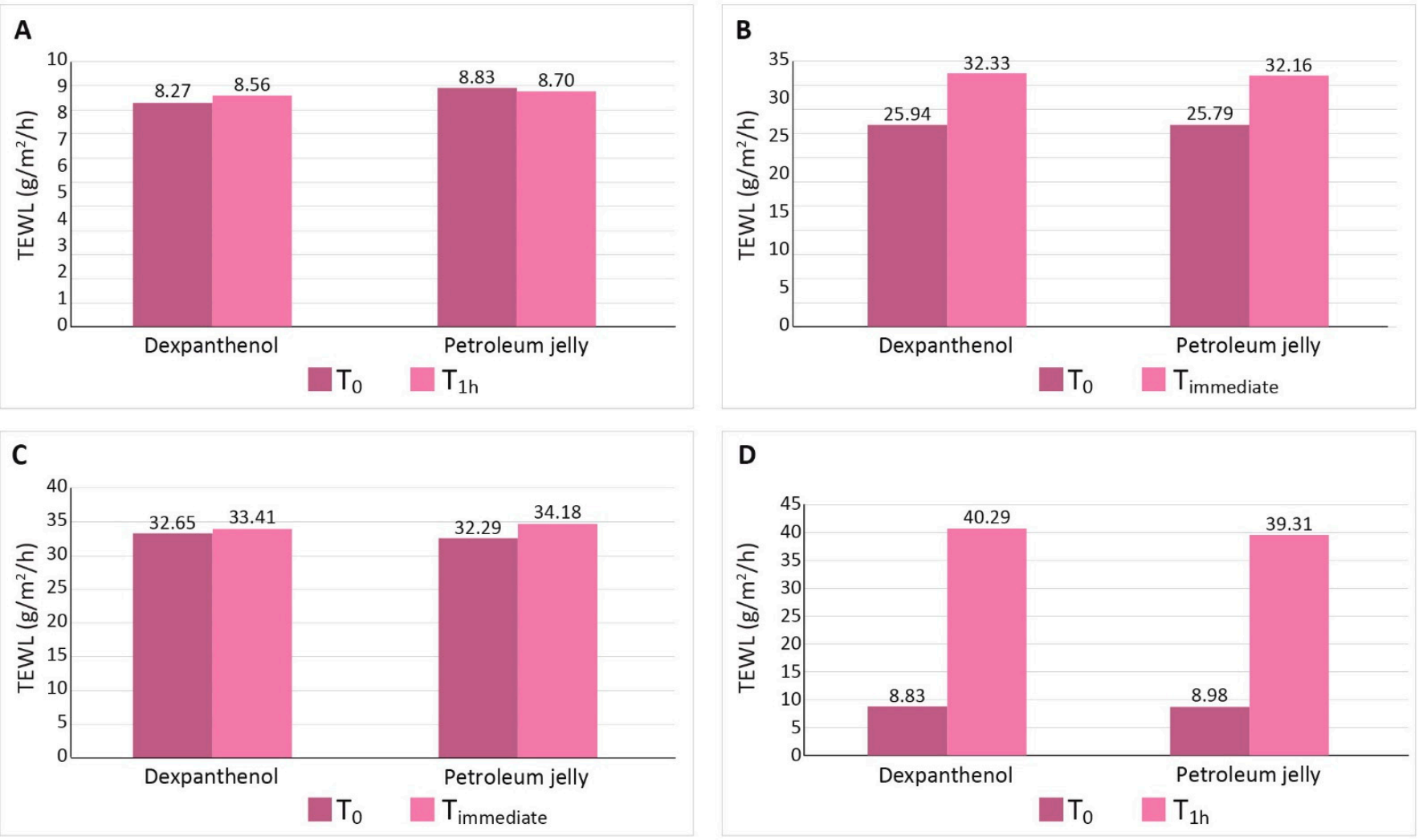

Figure 1. Transepidermal water loss (TEWL) $\left(\mathrm{g} / \mathrm{m}^{2} / \mathrm{h}\right)$ before (at T0) and post-procedure at baseline (T1) in both Dexpanthenol-containing products and control sides, before the application of test products (ITT). The products were tested in the same subjects, where the side (right or left) for test product application randomly selected. ITT: intention-to-treat group. In studies 1 and 4 (A,D), the post-procedure assessment (T1) was done $1 \mathrm{~h}$ after while in the studies 2 and 3 (B,C) it was done immediately after the procedure.

\subsection{Secondary Outcomes}

Secondary outcomes included clinical efficacy, safety, tolerability, and product acceptability.

\subsubsection{Clinical Efficacy—Dermatologists' Assessments}

Dermatologists assessed the clinical efficacy after $48 \mathrm{~h}$ (T48), and on D7, D14, and D21 (except for the laser depilation protocol, study (2), which did not include an assessment on day 14). The characteristics evaluated by the dermatologists varied depending on the procedure including hydration, softness, vitality, appearance of healthy skin, appearance of nourished skin, irritation/sensitivity, folliculitis, recovery after the procedure, peeling/renewal, and revitalization. The dermatologists scored each parameter on a scale ranging from 1 (worsened) to 3 (improved), or on a scale ranging from 1 (worst) to 5 (best).

\subsubsection{Clinical Efficacy-Subjects' Assessments}

Perceived efficacy: Subjects reported their perception of their own skin by evaluating hydration, softness, vitality, burning, itchiness, erythema, healthy appearance, nourished skin, irritation/sensitivity, recovery of the skin after the procedure, soothing effect, peeling/renewal, softness, and moisture of the skin after application. They answered the questions during the visits, giving scores from 1 (worst score) to 5 (best score).

\subsubsection{Safety and Tolerability}

Safety and tolerability were assessed by a dermatologist in all visits for all studies. Ophthalmologists (in the facial laser studies) and gynecologists (in the chemical peel study) made an initial assessment before the procedure (D0) and at the end of the studies (D21). 


\subsubsection{Product Acceptability}

The acceptability of Dexpanthenol-containing products was evaluated by participants through a subjective questionnaire at the end of the study after 21 days of product use. They were also asked to rank spreadability, ease of application, absorption, and stickiness either as "excellent", "good", "neutral", or "bad". Depending on the study, the assessment was adapted slightly for some product attributes (e.g., spreadability could be classified from "very easy/pleasant" to "very difficult/impossible to spread"). The participants" purchase intention was also collected.

\subsection{Statistical Analyses}

Parametric or non-parametric tests were used to compare variables with normal distribution or non-normal distributions, respectively. Within subjects' changes versus baseline were analyzed with paired $t$-test (normal distribution) or Wilcoxon test and Mann-Whitney (non-normal distribution). The baseline is described in this article as "T1", which means immediately after depilation and chemical peel and before the application of test products (studies (2) and (3)), or one hour after the facial laser procedures, before the application of the products (studies (1) and (4)). The baseline values of the primary outcome measures were compared with the results after $48 \mathrm{~h}$ (T48), and on D7, D14, and D21, except in the laser depilation protocol, which did not include an evaluation on D14. The within-subject comparison between test product and control was performed as well as the mean values of the products' attributes were compared at each time point.

Intention to treat (ITT) analyses and per-protocol (PP) analyses were conducted. The ITT population included all the randomized subjects, and the PP population refers only to those who completed the entire study.

Considering a dropout rate of $10 \%$, the targeted sample size ranged from 30 to 33 participants in each trial.

The protocols were registered at Plataforma Brasil [23] and were approved by an independent ethics committee (committee of Universidade São Francisco, São Paulo (SP), Brazil).

The study was sponsored by Bayer and conducted at Medcin Instituto da Pele.

\section{Results}

The number of subjects of ITT and PP analyses was almost identical. The PP populations had one subject less than ITT in all studies. Therefore, the results of ITT and PP analyses were similar. As the difference from the ITT to PP populations was only one subject in each study, we will present only the ITT analyses.

Table 2 shows the demographic characteristics of all included subjects.

Table 2. Population characteristics.

\begin{tabular}{cccccc}
\hline Study & \multicolumn{2}{c}{ Number } & Age, Years & Fitzpatrick Phototype & Gender \\
\hline & Screened & Included & Mean (Range) & (I, II, III, IV) & Female/Male \\
\hline 1 & 35 & 33 & $49(33-59)$ & I or II & Female \\
2 & 37 & 33 & $46(30-60)$ & I or II & Female \\
3 & 38 & 33 & $41(23-60)$ & I or II & Female \\
4 & 65 & 33 & $46(30-60)$ & I or II & Female \\
\hline
\end{tabular}

\subsection{Primary Outcomes}

\subsubsection{Recovery of Skin Integrity}

TEWL increased immediately (or within an hour) post-procedure in all studies, as seen by comparing $\mathrm{T} 0$ and $\mathrm{T} 1$ (Figure 1).

As expected, the ablative facial laser resurfacing (study (4)) was associated with the highest increase in the TEWL, while the non-ablative laser (study (1)) caused only a slight increase in the TEWL.

The ITT analysis showed that all products, Dexpanthenol-containing products (spray and cream) as well as petroleum jelly, decreased the TEWL, helping to recover skin barrier 
integrity after all the aesthetic procedures throughout the study (Figure 2). There was no difference in the effect of Dexpanthenol-containing products and control. The improvement of the skin barrier function was already significant after $48 \mathrm{~h}$ in studies (2)-(4), because the procedures caused an intense increase in TEWL, highlighting the impact of the procedure on the skin. The non-ablative laser did not impair the skin integrity (Figure 1) and the improvement reached statistical significance only on D21 (Figure 2).
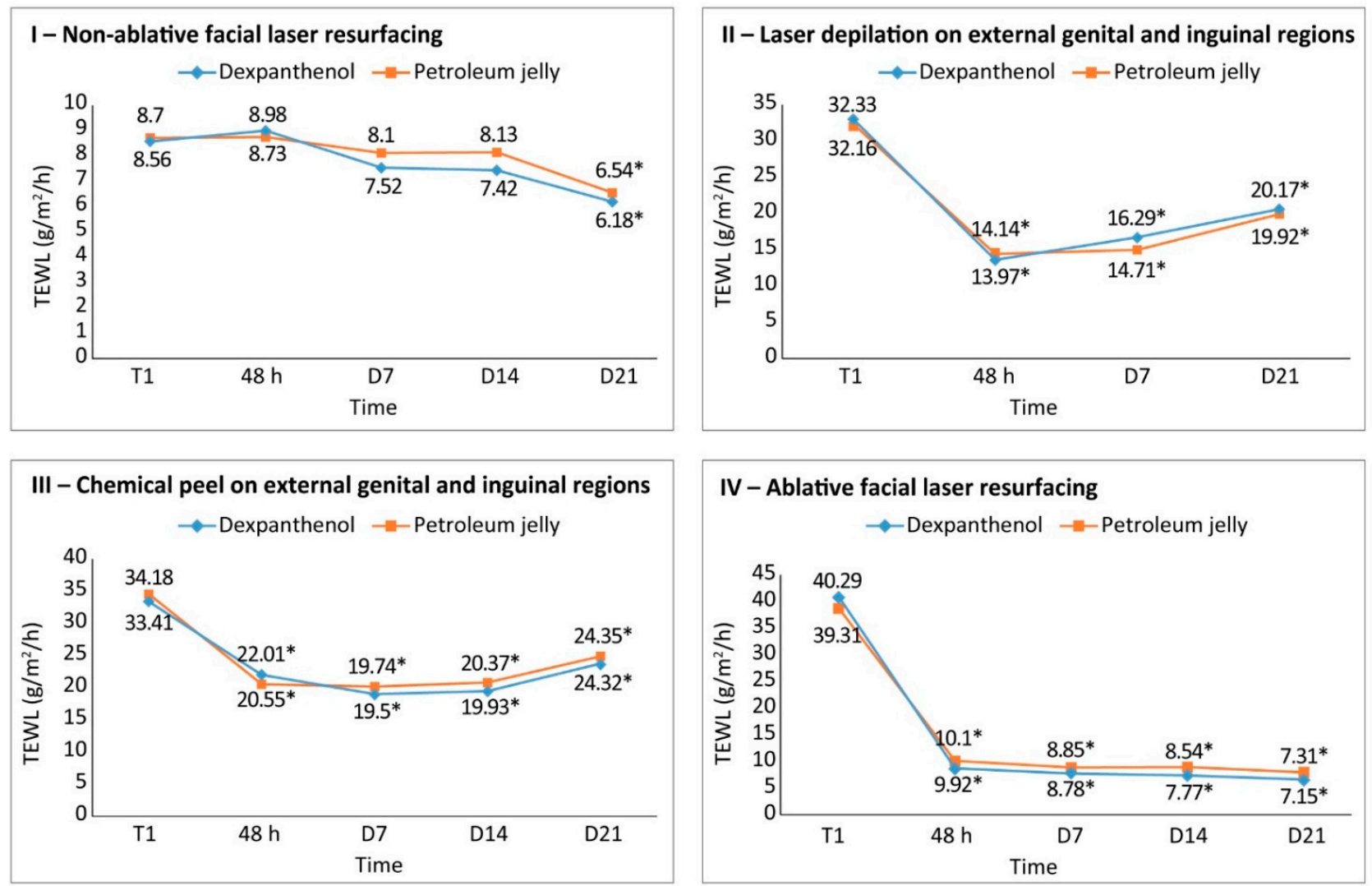

Figure 2. TEWL $\left(\mathrm{g} / \mathrm{m}^{2} / \mathrm{h}\right)$ at time points after each procedure (ITT); ${ }^{*} p<0.05 \mathrm{vs}$. T1. There were no significant differences between Dexpanthenol-containing products and Petroleum jelly. T1: baseline at either $1 \mathrm{~h}$ after the procedure (studies 1 and 4 ) and immediately after the procedure (studies 2 and 3).

\subsubsection{Reduction of Inflammation/Erythema}

Dermal temperature was assessed after non-ablative and ablative laser procedures. Photo records were taken with the thermographic camera at the beginning of the study (D0), one hour after the laser procedure (T1), at T48, on D7, D14, and D21.

In Study (1), after the non-ablative facial laser resurfacing, the skin inflammation decreased significantly after 21 days of treatment with either Dexpanthenol-containing spray or petroleum jelly $(p<0.05)$ (Table 3$)$.

After the ablative facial laser, which caused a more intense inflammation, a significant improvement from baseline was observed with Dexpanthenol-containing cream at all time points, while the improvement with petroleum jelly was significant only on D7 (Table 4). However, there was no statistical difference between the two products ( $p>0.05$ in all timepoints). 
Table 3. Mean change of thermographic values versus baseline after non-ablative facial laser (Study 1).

\begin{tabular}{|c|c|c|c|}
\hline Treatment & Timepoint & Change from Baseline (\%) & $p$ Value \\
\hline \multirow{4}{*}{ Dexpanthenol a } & $48 \mathrm{~h}$ & $-0.007 \%$ & 0.877 \\
\hline & Day 7 & $-0.51 \%$ & 0.198 \\
\hline & Day 14 & $-0.92 \%$ & 0.068 \\
\hline & Day 21 & $-1.24 \%$ & 0.010 \\
\hline \multirow{4}{*}{ Petroleum jelly (control) } & $48 \mathrm{~h}$ & $-0.08 \%$ & 0.858 \\
\hline & Day 7 & $-0.53 \%$ & 0.205 \\
\hline & Day 14 & $-0.95 \%$ & 0.058 \\
\hline & Day 21 & $-1.32 \%$ & 0.008 \\
\hline
\end{tabular}

Baseline (T1): $1 \mathrm{~h}$ after the procedure, before the application of the products. Student $t$-test was used for all the time points. Significance level: $5 \%$, shown in bold. ${ }^{\text {a }}$ Dexpanthenol-containing spray.

Table 4. Mean change of thermographic values versus baseline after ablative laser (Study 4).

\begin{tabular}{cccc}
\hline Treatment & Timepoint & Change from Baseline (\%) & $p$ Value \\
\hline & $48 \mathrm{~h}$ & $-1.57 \%$ & $\mathbf{0 . 0 0 5}$ \\
Dexpanthenol $^{\mathrm{a}}$ & Day 7 & $-1.98 \%$ & $\mathbf{0 . 0 0 3}$ \\
& Day $14 \mathrm{~b}$ & $-1.67 \%$ & $\mathbf{0 . 0 0 2}$ \\
& Day 21 & $-1.58 \%$ & $\mathbf{0 . 0 0 1}$ \\
Petroleum jelly (control) & $48 \mathrm{~h}$ & $-1.09 \%$ & 0.072 \\
& Day 7 & $-1.58 \%$ & $\mathbf{0 . 0 2 5}$ \\
& Day 14 & $-1.21 \%$ & 0.114 \\
\hline
\end{tabular}

Baseline (T1): $1 \mathrm{~h}$ after the procedure but before product application. ${ }^{\mathrm{a}}$ Dexpanthenol-containing cream. ${ }^{\mathrm{b}}$ Wilcoxon signed rank test (all the other time points were compared to baseline using the Student $t$-test). Significance level: $5 \%$, shown in bold.

\subsection{Secondary Outcomes}

\subsubsection{Clinical Efficacy}

Study 1. Non-Ablative Facial Laser

Clinical Efficacy: Dermatologists' Assessments.

Hydration, softness, vitality, healthy skin appearance, nourished skin, and irritation/sensitivity were assessed at T48, and on D7, D14, and D21, and compared with T1. The skin recovery after the procedure, peeling/renewal, and skin revitalization were not assessed at T1, as these refer to parameters related to the product.

Statistically significant improvement $(p<0.05)$ was observed in all parameters, at all time points in comparison to baseline at T1. There were no differences between the products.

Forty-eight hours after the non-ablative laser, skin hydration improved in $90.63 \%$ of the hemifaces treated with Dexpanthenol-containing spray and $81.25 \%$ of the hemifaces that received petroleum jelly.

A very good recovery/full recovery was found in $95.63 \%$ (25\% very good recovery $65.63 \%$ full recovery) for both products.

Clinical Efficacy: Subjects' Assessments.

A questionnaire about skin conditions was collected at T1, T48, D7, D14, and D21. The subjects evaluated their skin hydration, softness, vitality, burning, itching, erythema, peeling, irritation, healthy skin appearance, nourished skin appearance, calming effect, and freshness.

All these parameters improved over time with both products. Notably, subjects reported a rapid improvement of their skin irritation. Irritation was reported in 15.15\% and $21.21 \%$ of the hemifaces before Dexpanthenol-spray and petroleum jelly, respectively. However, at T48, this percentage decreased to $6.25 \%$ and $9.38 \%$ after the application of Dexpanthenol spray and petroleum jelly, respectively. From D14 onwards, 100\% of the subjects reported the absence of irritation/sensitivity with both products. 
Study 2: Laser Depilation around the External Genital Region (Skin Intimate Area)

Clinical Efficacy: Dermatologists.

The parameters of skin hydration, softness, erythema, vitality, healthy appearance, irritation/sensitivity, and prevention of folliculitis were assessed by a dermatologist at T48, D7, and D21 and compared with T1. However, skin recovery, peeling/renewal, skin revitalization, and calming effect were not assessed at $\mathrm{T} 1$, since they are only relevant after product application.

Both Dexpanthenol-containing spray and the comparator showed statistically significant improvement $(p<0.05)$ in the clinical parameters of skin hydration, softness, erythema, vitality, healthy skin appearance, and irritation/sensitivity at T48, D7, and D21. The improvement of the folliculitis was observed at D7 and D21, for both products $(p<0.05)$.

After $48 \mathrm{~h}$, the recovery of skin with Dexpanthenol-containing spray was rated by the dermatologists as "excellent" or "good" by $81.82 \%$; compared to $75.76 \%$ for the control product. These percentages increased over time and on D21 the recovery was considered "excellent" by $96.98 \%$ of the subjects for both products, while the remaining 3\% considered the recovery as "good" for both products.

Clinical Efficacy: Subjects' Assessments.

Subjects assessed their skin hydration, softness, vitality, burning, itchiness, erythema, rough/dryness sensation, healthy skin appearance, nourished skin appearance, and skin irritation/sensitivity. The assessments of T48, D7, and D21 were compared with baseline (T1).

Skin recovery after the procedure, freshness, calming effect, prevention of folliculitis, scaling, feeling of comfort, staining, and stickiness on the clothes were not evaluated at T1, as they are related to the product application.

Both treatments were evaluated positively regarding hydration, softness, vitality, burning, itching, erythema, rough/dry skin sensation, nourished skin appearance, irritation/sensitivity, healthy skin appearance, skin recovery after the procedure, freshness, calming effect, folliculitis, stained clothes, adherence/stickiness to clothes, feeling of comfort, quick recovery after the procedure, and scaling skin.

Regarding the prevention of folliculitis, an important aspect after laser depilation, at $\mathrm{T} 48,15.63 \%$ of the subjects classified their skin as "with no improvement", regardless of the treatment. This percentage decreased with both products to $6.25 \%$ on D7 and $3.13 \%$ on D21. On the other hand, the percentage of subjects who classified the improvement of folliculitis as "excellent" or "good" increased from 25\% at T48 for both to $71.85 \%$ and $75 \%$ on D21 for Dexpanthenol and control, respectively.

At T48, the feeling of comfort was rated as "absent" by 25\% (Dexpanthenol) and 21.88\% (control), but this percentage decreased to $3.13 \%$ on D21, for both products. Conversely, on D21, all the other subjects reported a feeling of comfort with either product. The stickiness to clothes was rated as "absent" in $96.88 \%$ of the side treated with Dexpanthenol and $90.63 \%$ of the sides treated with control.

Study 3: Chemical Peel in the Area around External Genitalia and Inguinal Area

Clinical Efficacy: Dermatologists' Assessments.

Clinical efficacy evaluations were performed by a dermatologist in the inguinal area and the area around the external genitalia immediately after the procedure (T1) and at T48, D7, D14, and D21 using the products. The parameters assessed were hydration, softness, erythema, vitality, healthy skin appearance, irritation/sensitivity, and prevention of folliculitis. Skin recovery after the procedure, peeling/renewal, skin revitalization, itching, and calming effect were not assessed at $\mathrm{T} 1$.

Both the Dexpanthenol-containing product and the comparator showed statistically significant improvement $(p<0.05)$ in the clinical parameters of hydration, softness, erythema, vitality, healthy skin appearance, and irritation/sensitivity after $48 \mathrm{~h}$. Softness, 
erythema, vitality, healthy skin appearance, and skin irritation/sensitivity significantly improved on D7, D14, and D21.

Because the procedure itself did not cause important scaling, the assessment of peeling/renewal, calming effect, and skin revitalization did not change at $\mathrm{T} 48$, for both products.

Clinical Efficacy: Subjects' Assessments.

The parameters hydration, softness, vitality, burning, itchiness, erythema, rough/dryness sensation, healthy skin appearance, nourished skin appearance, and irritation/sensitivity were assessed at baseline (T1) and compared to T48, D7, D14, and D21. The recovery of the skin after the procedure, freshness, calming effect, prevention of folliculitis, peeling, comfort, and the products staining and stickiness on clothes, were not assessed at T1, as they are related to the products.

Except for peeling (scaling) and folliculitis, all the parameters were evaluated positively by the subjects.

The assessment of peeling/renewal by the subjects was aligned with the dermatologists' assessment. Since the procedure did not induce notable scaling, this parameter did not change after the application of products. The same occurred with the self-assessment of folliculitis, as the participants did not have folliculitis at start of the study.

Study 4: Dexpanthenol-Containing Emollient Cream after Facial Ablative Laser

Clinical Efficacy: Dermatologists' Assessments.

Hydration, softness, vitality, healthy skin appearance, nourished skin, and irritation/sensitivity, erythema, and smoothness were assessed at T48, D7, D14, and D21, and compared to baseline T1 (one hour after the procedure, before the application of the products). Skin recovery after the procedure, peeling/renewal, and revitalization were not evaluated at $\mathrm{T} 1$.

Both products showed statistically significant improvement $(p<0.05)$ in clinician's assessment of skin hydration, softness, irritation/sensitivity, erythema, and smoothness after $48 \mathrm{~h}$. Erythema improved in 100\% of the hemifaces after $48 \mathrm{~h}$, with both products.

All the parameters improved in the subsequent time points, with no difference between the products.

The procedure itself did not induce significant flaking, and therefore the assessment of peeling/renewal and revitalization did not change from $48 \mathrm{~h}$ to D7, D14, and D21, similarly for both products.

Clinical Efficacy: Subjects' Assessments.

Hydration, softness, vitality, burning, pruritus, erythema, healthy appearance, nourished skin appearance, and irritation/sensitivity were assessed at T48, D7, D14, and D21 and compared to T1. Recovery of the skin after the procedure, soothing effect, peeling/renewal, softness, and the moisture of the skin after application were not assessed at $\mathrm{T} 1$ because such characteristics are related to the application of the products.

All the parameters improved over time, particularly the irritation/sensitivity, burning sensation, and erythema. Irritation was considered intense by $21.21 \%$ in the hemifaces before receiving the products and moderate by $39.39 \%$ in the hemifaces before Dexpanthenol cream and $42.42 \%$ before the petroleum jelly. After $48 \mathrm{~h}, 84.38 \%$ of the hemifaces treated with Dexpanthenol-containing cream and $75 \%$ of the hemifaces treated with petroleum jelly were evaluated as without irritation, and the improvement continued over time. On D7, $96.77 \%$ of the hemifaces of both products were assessed as without irritation. The burning sensation was present in almost all participants; after $48 \mathrm{~h}$, it was considered "absent" in $87.10 \%$ and $80.65 \%$ of the hemifaces treated with Dexpanthenol cream and petroleum jelly, respectively. This percentage increased for both groups with the same proportion: $96.77 \%$ on D7 and 100\% on D14 and D21, with no differences between the products.

After $48 \mathrm{~h}$, the subjects rated erythema as "absent" in $68.75 \%$ and $65.63 \%$ of the hemifaces treated with Dexpanthenol-containing emollient cream and with control, respectively. This percentage increased to $93.55 \%$ for both products on D7, and to 100\% on D14 and D21 for Dexpanthenol-containing emollient and to $96.77 \%$ on D14 and 100\% on D21 for Control. 


\subsubsection{Safety and Tolerability}

The safety assessments were conducted by dermatologists in all four studies. Studies (1) and (4) (facial procedures) included an ophthalmological evaluation on the preprocedure visit (D0) and D21. Study (3) (chemical peel) included a gynecological assessment on D0 and D21, and study (2) (laser depilation) included a gynecological evaluation on D0.

The safety and tolerability profiles of Dexpanthenol-containing products and petroleum jelly were similar. There was no serious adverse event in any of the studies, neither ophthalmologic nor gynecologic.

There were three adverse events in trial (3): two subjects had pruritus and one presented gastroenterocolitis. The correlation with the test product was considered "not probable" (one case of pruritus) and the other adverse events were considered unrelated to the test product.

In trial (2), 13 subjects had erythema; one had edema, one presented with peeling, three reported burning, and one reported itching. All the adverse events reported were considered expected following the procedure and unrelated to the test product. On D21, there were no adverse events reported.

In study (1), there was no adverse event related to the product and no ophthalmologic adverse event.

In study (4), there were six reported adverse events, with five of them expected following the procedure and considered not related to the test product (pruritus, burning, peeling, roughness) and one which was neither related to the procedure nor the test product (anxiety, sickness).

\subsection{Acceptability}

The attributes of Dexpanthenol-containing products were classified within the two best scores (e.g., "excellent" and "good") by the majority of the subjects. Table 5 summarizes the results of acceptability in the four studies.

Table 5. Acceptability: assessment of physical attributes and purchase intention.

\begin{tabular}{|c|c|c|c|c|}
\hline Study & $\begin{array}{c}\text { 1-Non-Ablative Laser } \\
\text { Resurfacing } \\
(n=31)\end{array}$ & $\begin{array}{l}\text { 2-Laser Depilation on } \\
\text { External Genital/Inguinal } \\
\text { Region } \\
(n=32)\end{array}$ & $\begin{array}{l}\text { 3-Chemical Peel on } \\
\text { External Genital/Inguinal } \\
\text { Region } \\
(n=31)\end{array}$ & $\begin{array}{l}\text { 4-Ablative Facial Laser } \\
\text { Resurfacing } \\
(n=31)\end{array}$ \\
\hline $\begin{array}{c}\text { Dexpanthenol-Containing } \\
\text { Formulation }\end{array}$ & Spray & Spray & Spray & Cream \\
\hline \multicolumn{5}{|c|}{ Attribute } \\
\hline $\begin{array}{c}\text { Smell } \\
\text { (\% excellent and good) }\end{array}$ & $54.84 \%$ & $50 \%$ & $38.71 \%$ * & $55.17 \%$ \\
\hline $\begin{array}{c}\text { Application } \\
\text { (\%very easy and easy) }\end{array}$ & $87.09 \%$ & $68.76 \%$ & $38.71 \%$ * & $82.76 \%$ \\
\hline $\begin{array}{c}\text { Spreadability } \\
\text { (\%very easy and easy) } \\
\text { Stickiness }\end{array}$ & $87.1 \%$ & $75 \%$ & $83.87 \%$ & $82.76 \%$ \\
\hline $\begin{array}{c}\text { (\% without stickiness and } \\
\text { lightly sticky) }\end{array}$ & $67.74 \%$ & $75 \%$ & $74.19 \%$ & $72.42 \%$ \\
\hline $\begin{array}{c}\text { Absorption } \\
\text { (\% very easy/pleasant } \\
\text { and easy) }\end{array}$ & $83.87 \%$ & $78.13 \%$ & $83.87 \%$ & $75.86 \%$ \\
\hline $\begin{array}{l}\text { Velvety touch } \\
\text { (\%Very velvety and velvety) }\end{array}$ & NA & NA & NA & $90.32 \%$ \\
\hline $\begin{array}{l}\text { Purchase intention } \\
\text { (\% certainly and probable) }\end{array}$ & $74.2 \%$ & $81.25 \%$ & $74.19 \%$ & $89.65 \%$ \\
\hline
\end{tabular}

* $48.39 \%$ of users assessed the smell and application as "neutral". NA: not assessed.

When the smell was not classified as "excellent" or "good", it was usually classified as "neutral". Only a few subjects considered the smell unpleasant. In study (3), although only $38.71 \%$ classified the smell as "excellent" or "good", $48.39 \%$ considered it "neutral". The same happened regarding the application, while $38.71 \%$ considered it very easy/easy, $48.39 \%$ considered it "not easy nor difficult". Acceptability and purchase intention were favorable for both spray and cream formulations (Table 5). 


\section{Discussion}

Laser and chemical peels are among the most popular procedures in dermatological clinics $[5,6]$. There is a need for effective post-procedural care. Additionally, dermatologists usually look for a fragrance-free and gentle product (to decrease the risk of allergies) to support the recovery of the skin barrier without being occlusive, sticky, and greasy, which could slow down the skin repair process and induce bacterial infection. Petrolatum derivatives are commonly used for this purpose due to their inert property. However, dermatologists currently prefer products that not only protect the skin, but are also able to repair minor damage and protect from transepidermal water loss, enabling the skin to recover by producing natural moisturizing factors (NMFs) and lipids by itself. In addition, the greasy feel of petroleum jelly limits its use post aesthetic procedures, as they are not well accepted by subjects, particularly on the face.

Dexpanthenol-containing products stand as an alternative to petroleum jelly. Indeed this molecule provides a dual-action benefit for subjects in need of skin care and/or wound healing by improving skin hydration, as well as promoting epidermal regeneration [24].

Another concern usually brought by clinicians when using petrolatum derivatives is their occlusiveness, which disturbs the skin barrier recovery and may cause acneiform lesions due to the clogged sebaceous glands and bacteria proliferation [25]. This is a frequent side effect with more occlusive substances, which is clearly undesired by dermatologists. In our studies, no acneiform lesions were observed with Dexpanthenol-containing products.

Dexpanthenol-containing products, either in spray or cream formulation, helped the skin recovery process, by maintaining the integrity of the skin barrier, as demonstrated with the TEWL and thermal camera assessments, thus confirming previous studies [26,27]. In study (4), there was a significant improvement from baseline with Dexpanthenol-containing cream in all time points, while a significant improvement with petroleum jelly was seen only on D7. Although the treatments were not different at each time point, there is a clear trend for superiority of Dexpanthenol-containing cream in improving the erythema after ablative laser, with a faster and sustained effect compared to the positive control.

The clinical efficacy of Dexpanthenol-containing products was confirmed by both dermatologists and subjects. They improved skin hydration, decreased irritability/sensitivity, and provided a feeling of comfort after the procedures.

Although the efficacy of Dexpanthenol-containing products and petroleum jelly were similar, the physical attributes are important particularly to customers of aesthetic clinics, who may demand products with more pleasant characteristics, instead of the waxy feel of petroleum jelly, which may be inconvenient after some procedures. Dexpanthenolcontaining spray and cream are fragrance- and colorant-free. The spray formulation is water-based, non-irritant, and non-comedogenic. Consequently, their acceptability was high among the users, in terms of smell, spreadability, and pleasantness.

To the best of our knowledge, studies (2) and (3) were the first to evaluate the effect of Dexpanthenol-containing products after procedures around the external genitalia (chemical peel and depilation). The light and fluid texture of Dexpanthenol-containing spray are adequate for aftercare in this area, which requires products that do not stick on clothes. In addition, the spray formulation allows a touchless application, reducing the risk of skin irritation due to friction.

Our studies have the following limitations: a small sample size, the single-blind design, the assessment by the treating dermatologists, the short trial duration, no collection of the subjective assessment of the physical properties of the control (petroleum jelly), and the limited ethnic diversity.

\section{Conclusions}

Dexpanthenol-containing products (spray and cream) displayed a similar efficacy and safety compared to the positive control (petroleum jelly) in aesthetic post-procedural care. Both test products and control helped to restore the skin barrier integrity and reduced inflammation (erythema) after laser aesthetic procedures and chemical peel, with good 
safety and tolerability profile. In addition, Dexpanthenol-containing products were well appreciated by the participants from a sensory perspective, addressing a patient need to have a product pleasant to be used following such aesthetic procedures. Consequently, the study suggests that these products are adequate for aesthetic post-procedural care and are appreciated from a sensorial perspective by both study participants and dermatologists.

Author Contributions: Conceptualization, F.A.S.A., M.C.d.S. and J.M.C.; methodology, F.A.S.A., M.C.d.S. and J.M.C.; formal analysis, F.A.S.A., M.C.d.S., S.T., E.P. and J.M.C., investigation F.A.S.A., resources, F.A.S.A., M.C.d.S. and J.M.C.; data curation, F.A.S.A., M.C.d.S. and J.M.C.; writingoriginal draft preparation, J.M.C., S.T. and E.P.; writing-review \& editing, F.A.S.A., M.C.d.S., S.T., E.P. and J.M.C.; visualization; J.M.C., S.T. and E.P.; supervision, M.C.d.S. and J.M.C.; project administration, F.A.S.A.; funding acquisition, M.C.d.S. and J.M.C. All authors have read and agreed to the published version of the manuscript.

Funding: Bayer Consumer Health (Brazil) sponsored the four studies and funded the editorial assistance provided by Monica Kayo, MD (Triple Check Consulting).

Institutional Review Board Statement: The study was conducted according to the guidelines of the Declaration of Helsinki, and approved by the Ethics Committee of Universidade São Francisco, São Paulo, SP, Brazil. Studies 1 and 2 were approved on 18 December 2019 (number 03736818.0.0000.5514 and 03736918.9.0000.5514). Study 3 was approved on 14 December 2018 (number 03737118.9.0000.5514), and Study 4 was approved on 11 February 2019 (number 05987119.2.0000.5514). The approval is registered and published at Plataforma Brasil (https: / / plataformabrasil.saude.gov.br/login.jsf (accessed on 10 August 2021)).

Informed Consent Statement: Written informed consent was obtained from all subjects involved in the study.

Data Availability Statement: Not applicable.

Conflicts of Interest: FASA has received research grants and honoraria for advisory board participation or speaker activities from Aché, Galderma, Libbs, and Loreal. JMC and MCS are employees of Bayer Consumer Health (Brazil). ST and EP are employees of Bayer Consumer Care AG (Switzerland).

\section{References}

1. Ong, M.W.S.; Bashir, S. Fractional laser resurfacing for acne scars: A review. Br. J. Dermatol. 2012, 166, 1160-1169. [CrossRef]

2. Kaushik, S.B.; Alexis, A.F. Nonablative Fractional Laser Resurfacing in Skin of Color: Evidence-based Review. J. Clin. Aesthet. Dermatol. 2017, 10, 51.

3. Narins, R.; Narins, D. Nonablative skin resurfacing. Aesthet. Surg. J. 2004, 24, 281-284. [CrossRef] [PubMed]

4. Manstein, D.; Herron, G.; Sink, R.; Tanner, H.; Anderson, R. Fractional photothermolysis: A new concept for cutaneous remodeling using microscopic patterns of thermal injury. Lasers Surg. Med. 2004, 34, 426-438. [CrossRef] [PubMed]

5. Devgan, L.; Singh, P.; Durairaj, K. Minimally Invasive Facial Cosmetic Procedures. Otolaryngol. Clin. N. Am. 2019, 52, 443-459. [CrossRef]

6. Rendon, M.I.; Berson, D.S.; Cohen, J.L.; Roberts, W.E.; Starker, I.; Wang, B. Evidence and considerations in the application of chemical peels in skin disorders and aesthetic resurfacing. J. Clin. Aesthet. Dermatol. 2010, 3, 32-43. [PubMed]

7. Samargandy, S.; Raggio, B.S. Skin Resurfacing Chemical Peels. In StatPearls [Internet]; StatPearls Publishing: Treasure Island, FL, USA, January 2021; [Updated 25 July 2021]. Available online: https:/ / www.ncbi.nlm.nih.gov/books / NBK547752 (accessed on 27 August 2021).

8. Garg, V.; Sinha, S.; Sarkar, R. Glycolic acid peels versus salicylic-mandelic acid peels in active acne vulgaris and post-acne scarring and hyperpigmentation: A comparative study. Dermatol. Surg. 2009, 35, 59-65. [CrossRef]

9. Yokomizo, V.; Benemond, V.; Chisaki, C.; Benemond, P. Chemical peels: Review and practical applications. Surg. Cosmet. Dermatol. 2013, 5, 56-68.

10. Castillo, D.E.; Keri, J.E. Chemical Peels in the Treatment of Acne: Patient Selection and Perspectives. Clin. Cosmet. Investig. Dermatol. 2018, 11, 365-372. [CrossRef]

11. Sarkar, R.; Ghunawat, S.; Garg, V.K. Comparative Study of 35\% Glycolic Acid, 20\% Salicylic-10\% Mandelic Acid, and Phytic Acid Combination Peels in the Treatment of Active Acne and Postacne Pigmentation. J. Cutan. Aesthet. Surg. 2019, 12, 158. [CrossRef]

12. Gorski, J.; Proksch, E.; Baron, J.M.; Schmid, D.; Zhang, L. Dexpanthenol in wound healing after medical and cosmetic interventions (Postprocedure wound healing). Pharmaceuticals 2020, 13, 138. [CrossRef] [PubMed]

13. Morales-Burgos, A.; Loosemore, M.P.; Goldberg, L.H. Postoperative wound care after dermatologic procedures: A comparison of 2 commonly used petrolatum-based ointments. J. Drugs Dermatol. 2013, 12, 163-164. [PubMed] 
14. Ebner, F.; Heller, A.; Rippke, F.; Tausch, I. Topical use of dexpanthenol in skin disorders. Am. J. Clin. Dermatol. 2002, 3, 427-433. [CrossRef] [PubMed]

15. Proksch, E.; de Bony, R.; Trapp, S.; Boudon, S. Topical use of dexpanthenol: A 70th anniversary article. J. Dermatol. Treat. 2017, 28, 766-773. [CrossRef] [PubMed]

16. Slyshenkov, V.S.; Rakowska, M.; Moiseenok, A.G.; Wojtczak, L. Pantothenic acid and its derivatives protect ehrlich ascites tumor cells against lipid peroxidation. Free Radic. Biol. Med. 1995, 19, 767-772. [CrossRef]

17. Heise, R.; Schmitt, L.; Huth, L.; Krings, L.; Kluwig, D.; Katsoulari, K.V.; Steiner, T.; Hölzle, F.; Baron, J.M.; Huth, S. Accelerated wound healing with a dexpanthenol-containing ointment after fractional ablative $\mathrm{CO}_{2}$ laser resurfacing of photo-damaged skin in a randomized prospective clinical trial. Cutan. Ocul. Toxicol. 2019, 38, 274-278. [CrossRef]

18. Czarnowicki, T.; Malajian, D.; Khattri, S.; da Rosa, J.C.; Dutt, R.; Finney, R.; Dhingra, N.; Xiangyu, P.; Xu, H.; Estrada, Y.D.; et al. Petrolatum: Barrier repair and antimicrobial responses underlying this "inert" moisturizer. J. Allergy Clin. Immunol. 2016, 137, 1091-1102. [CrossRef] [PubMed]

19. Bennani, I.; Lopez, R.; Bonnet, D.; Prevot, G.; Constantin, A.; Chauveau, D.; Paul, C.; Livideanu, C.B. Improvement of Microstomia in Scleroderma after Carbon Dioxide Laser Treatment. Case Rep. Dermatol. 2016, 8, 142-150. [CrossRef]

20. Wall, T. Current Concepts: Laser Treatment of Adult Vascular Lesions. Semin. Plast. Surg. 2007, 21, 147-158. [CrossRef]

21. Ghadially, R.; Halkier-Sorensen, L.; Elias, P.M. Effects of petrolatum on stratum corneum structure and function. J. Am. Acad. Dermatol. 1992, 26, 387-396. [CrossRef]

22. Fluhr, J.W.; Feingold, K.R.; Elias, P.M. Transepidermal water loss reflects permeability barrier status: Validation in human and rodent in vivo and ex vivo models. Exp. Dermatol. 2006, 15, 483-492. [CrossRef] [PubMed]

23. Plataforma Brasil. Available online: https:// plataformabrasil.saude.gov.br/login.jsf (accessed on 1 March 2021).

24. Stettler, H.; Crowther, J.M.; Brandt, M.; Lu, B.; Boxshall, A.; de Salvo, R.; Laing, S.; Hennighausen, N.; Bielfeldt, S.; Blenkiron, P. Targeted dry skin treatment using a multifunctional topical moisturizer. Int. J. Cosmet. Sci. 2020, 43, 191-200. [CrossRef]

25. Frankel, E.B. Acne Secondary to White Petrolatum Use. Arch. Dermatol. 1985, 121, 589-590. [CrossRef]

26. Gehring, W.; Gloor, M. Effect of topically applied dexpanthenol on epidermal barrier function and stratum corneum hydration: Results of a human in vivo. Arzneim.-Forsch./Drug Res. Ed. 2000, 50, 659-663. [CrossRef]

27. Proksch, E.; Nissen, H.P. Dexpanthenol enhances skin barrier repair and reduces inflammation after sodium lauryl sulphateinduced irritation. J. Dermatol. Treat. 2002, 13, 173-178. [CrossRef] [PubMed] 